
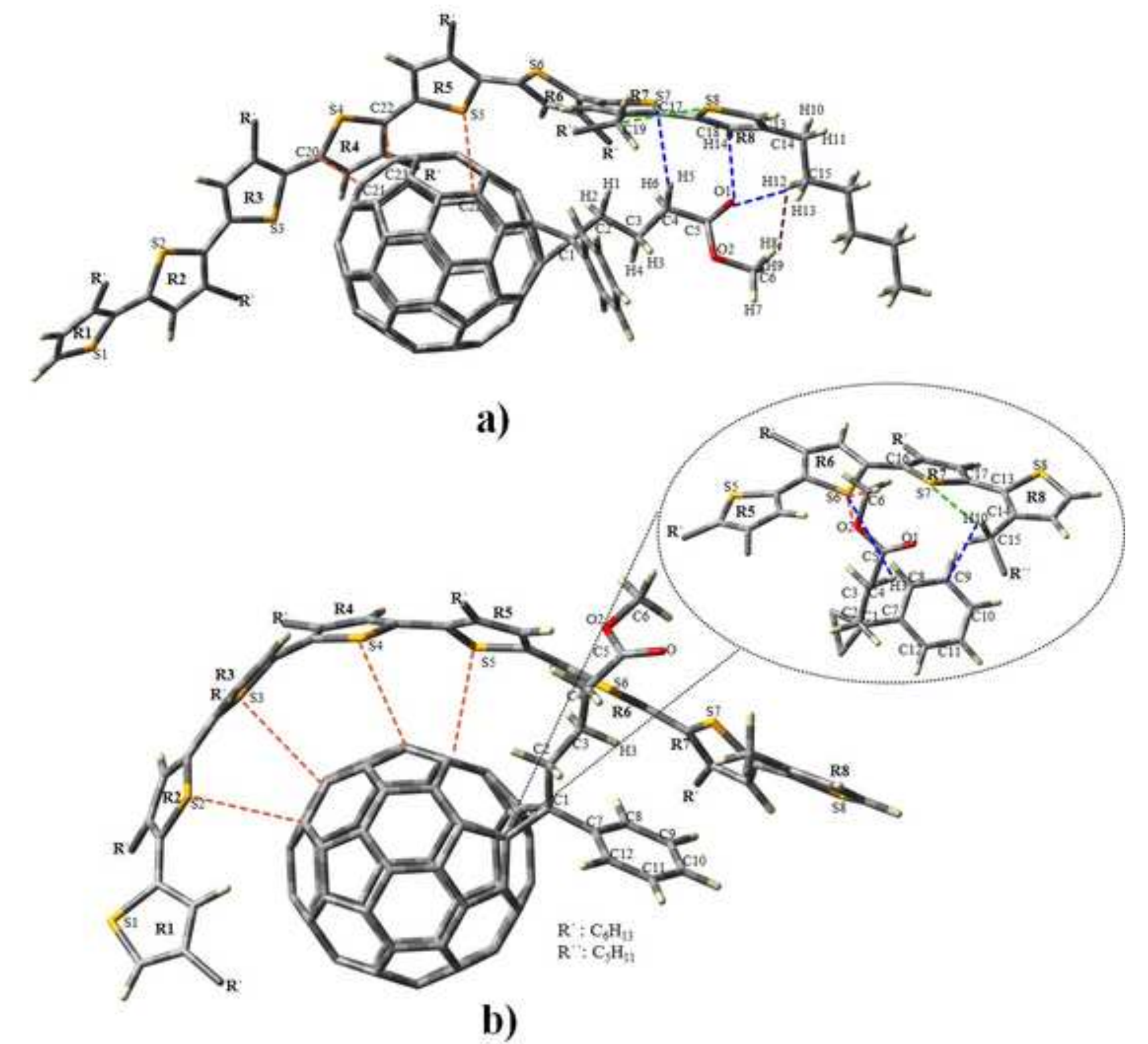

b) 


\title{
A QTAIM Topological Analysis of the P3HT-PCBM Dimer
}

\author{
Juan I. Rodríguez, ${ }^{1 *}$ Chérif F. Matta, ${ }^{2}$ Emilbus A. Uribe, ${ }^{3}$ Andreas W. Götz, ${ }^{4}$ \\ F.L. Castillo-Alvarado, ${ }^{1}$ and Bertha Molina-Brito ${ }^{5}$ \\ ${ }^{1}$ Escuela Superior de Física y Matemáticas, Instituto Politécnico Nacional, Edificio 9, U.P. A.L.M., \\ Col. San Pedro Zacatenco, C.P. 07738, México D.F., México. \\ ${ }^{2}$ Mount Saint Vincent University, Halifax, Canada, B3M 2J6. \\ ${ }^{3}$ Departamento de Ciencias Básicas, Universidad Santo Tomás, Carrera 9 No. 51-11, Bogotá, \\ Colombia. \\ ${ }^{4}$ San Diego Supercomputer Center, University of California San Diego, 9500 Gilman Drive, La \\ Jolla, CA, 92093-0505, USA., \\ ${ }^{5}$ Facultad de Ciencias, Universidad Nacional Autónoma de México, Apartado Postal 70-646.
}

\begin{abstract}
In order to cast some light onto the nature of the chemical bonding between a 8-unit oligomer of the poly(3-hexylthiophene) (P3HT) and the fullerene derivative [6,6]-phenyl-C 61 -butyric acid methyl ester (PCBM) in the two stables isomers reported recently [I. Gutiérrez-González, B. Molina-Brito, A.W. Götz, F.L. Castillo-Alvarado, J.I. Rodríguez, Chem. Phys. Lett. 612, 234 (2014)], we have performed a Bader's quantum theory of atoms in molecules (QTAIM) analysis. According to QTAIM, no covalent bonds are formed between P3HT and PCBM, and hydrogen and stacking interactions account for about $90 \%$ and $10 \%$ of the total number of bonds between P3HT and PCBM, respectively.
\end{abstract}

Keywords: Photovoltaic cells, weak bonding interactions, hydrogen bonding, topological analysis of the electron density, Bader's quantum theory of atoms in molecule (QTAIM).

* Corresponding author: Juan I. Rodríguez. E-mail: juan@esfm.ipn.mx; jirodriguezh@ipn.mx 


\section{Introduction}

Organic or plastic solar cells represent a technology that might increase the green worldwide energy production. Their low-cost, flexibility and transparency is poised to make it possible for these devices to be a massive-use technology as long as its efficiency is increased. Worldwide research focused on increasing such an efficiency is ongoing [1-2]. Among the most efficient plastic solar cells $(\eta \approx 5-7 \%)$ reported to date are the so called bulk-heterojunction (BHJ) cells in which the active layer is a mix of the polymer poly(3-hexylthiophene) (P3HT) in the head-to-tail arrangement (SCHEME I(a)) and the fullerene derivative [6,6]-phenyl-C 61 -butyric acid methyl ester (PCBM) (SCHEME I(b)) [3].

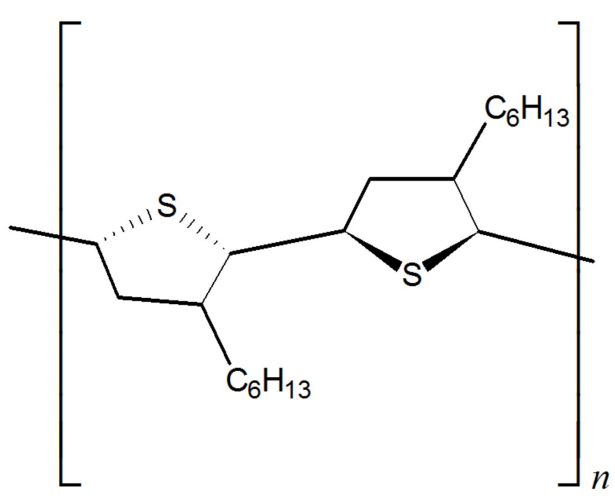

(a) Poly(3-hexylthiophene) (P3HT)

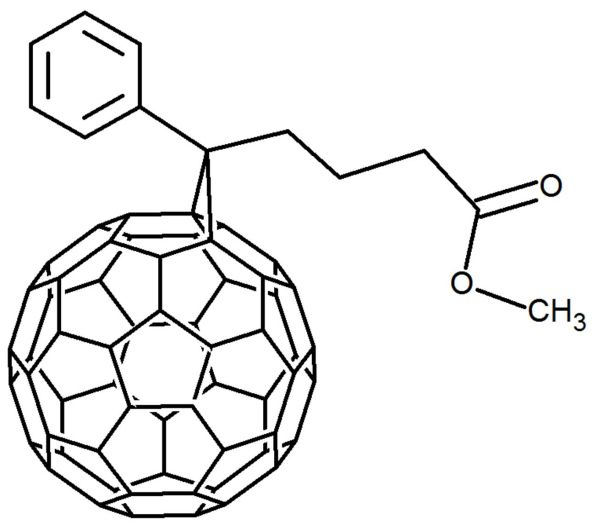

(b) $[6,6]$-phenyl-C61-butyric acid methyl ester (PCBM)

\section{SCHEME I}

Interaction between the electron donor polymer (P3HT) and acceptor (PCBM) at the P3HTPCBM interface, where the exciton formation/dissociation/recombination mainly develops, plays a crucial role on determining the active layer electronic and optical properties and the cell efficiency [427]. Yet a complete understanding of how the P3HT and the PCBM interact at the molecular level remains to be elucidated. What type of chemical bonding links the two components, what kind of structure they form, and how these processes influence the active layer properties are among the key 
questions to be answered. This paper will shed some light in this direction.

In order to cast some light onto how the PCBM and P3HT interact at the molecular level we recently performed Kohn-Sham density functional theory (DFT) calculations on the supramolecular dimer formed by a 8-unit oligomer (8-mer) of P3HT and the PCBM in the gas phase reporting two stable P3HT-PCBM dimer isomers [27]. In both isomers, the P3HT tends to "embrace" the PCBM adopting a U-shape due mainly to van der Waals interactions [27]. The influence of the dimer structure on the optical properties is in qualitative agreement with the experimental blue shift of the absorption spectrum peaks. [27] As expected, no covalent bonds are formed between the P3HT and the PCBM as based on the analysis of the interatomic distances. The shortest interatomic distance between atoms on the P3HT and PCBM is equal to 2.08 and 2.27A for isomer 1 and isomer 2, respectively [27]. The van der Waals interaction, taken into account via the Grimme dispersion correction [28-29], played a key role for the P3HT to adopt such a U-shape surrounding the PCBM.

Noncovalent interactions involving hydrogen atoms may be expected to play a role in stabilizing the P3HT-PCBM dimer but this must be shown and quantified based on quantum chemical/computational evidence which is a main goal of this work. It is well known that it is not always possible to quantify these interactions directly from an electronic structure calculation. Bader's Quantum Theory of Atoms in Molecules (QTAIM) [30,31] has been used for describing different types of chemical bonding. In particular, QTAIM has previously been used to quantify stacking and hydrogen-bonded interactions in some representative complexes [30-35]. Interactions energies were correlated with the values of the electron density, the potential energy density, and the Laplacian of the density at the bond critical points (CPs) between dimers. [33-36]

In this work we performed a topological analysis of the electron density in accordance with Bader's QTAIM [30,31] to cast light onto the nature of the chemical bonding between the P3HT and PCBM in the supramolecular P3HT-PCBM complex. Both Bader properties and topology of the 
electron density analysis were performed using the high-performing method developed by us especially to study large systems [37-38] implemented in the Amsterdam Density Functional (ADF 2014) package [39-41].

\section{Computational Details}

For each of the P3HT-PCBM dimer isomers, a self consistent field (SCF) single point (SP) DFT calculation followed by a QTAIM calculation was performed at the stable geometries reported in our previous work [27] (see Figure 1). All DFT and QTAIM calculations were performed with ADF package [39-41]. The (generalized-gradient-approximation) Perdew-Burke-Ernzerhof (PBE) exchangecorrelation (xc) functional [42] and a triple- $\zeta$ polarized (TZP) Slater basis sets from the ADF basis library were used. The ADF integration parameter was set to 6 in order to ensure small errors in solving the Kohn-Sham DFT equations and computing the QTAIM properties [37-41]. The QTAIM calculations were performed using our ultrafast method [37-38] which was implemented in ADF [3741]. ADF default settings for the SCF procedure and property calculations were used [39-41].

\section{Results and Discussion}

Figure 1 shows the two stable isomer structures (relative energy $3.13 \mathrm{eV}$, Isomer 1 is the lowest-energy isomer) of the P3HT-PCBM dimer reported in detail in our original study [27], thus only the most relevant features of these structures for the present study are introduced here. Adjacent thiophene rings in these U-shaped P3HT undergo a torsion $\left(\sim 15^{\circ}\right)$ of the P3HT thiophene rings around an axes "parallel” to the backbone. These rotations happen in a alternate way: one ring is rotated clockwise and the adjacent ring counter clockwise and so on. The dihedral angles between adjacent thiophene rings in the P3HT chain in the dimer can be seen in Figure 2 (which was reproduced from reference [27]). Notice that R7 in dimer Isomer 2 (I2) gets into a head-head position with R6 breaking the global head- 
tail arrangement between two adjacent rings in the P3HT chain (breaking so the local translational symmetry). In Isomer 1 (I1), however, the head-tail arrangement of the P3HT thiophene rings holds. Thus it can be considered that the destruction of order on the P3HT chain is more drastic for I2. The interaction energy ( $E^{\text {interaction }}=E^{\text {dimer }}-E^{P 3 H T}-E^{P C B M}$ ) is equal to $356.7 \mathrm{~kJ} / \mathrm{mol}$ and $54.6 \mathrm{~kJ} / \mathrm{mol}$ for Isomer 1 and Isomer 2, respectively (basis set superposition error correction was not considered since the accurate determination of the binding energies is not the goal of this work).

According to QTAIM, there is a bond critical point (BCP) between each pair of chemical bonded atoms in a molecule. [30-31,38] Due to the supramolecular nature of the P3HT-PCBM dimer considered here, the BCPs can be classified into two types: intramolecular and “intermolecular” BCPs. There is an intramolecular BCP between each pair of bonded atoms belonging to either of the monomers (P3HT or PCBM); there is an intermolecular BCP (interBCP) between each pair of chemically bonded atoms in which one atom is on the P3HT and the other on the PCBM. As stated above, in this work we are interested in applying QTAIM for casting some light onto the nature of the bonding between the P3HT and PCBM. Thus we will focus on the bond information coming from the intermolecular BCPs. Most the bond information from the intramolecular BCPs is quite predictable since it is similar to that well known information obtained on organic covalent bonded molecules (see discussion below) [30-31].

In order to better determine the role of each chemical group in the system, we have analyzed the interBCP bond information separating it into the contribution of $(i)$ the $\mathrm{P} 3 \mathrm{HT}$ side chains $(\mathrm{SC})=\mathrm{C}_{6} \mathrm{H}_{13}$, (ii) the P3HT backbone (BB), (iii) the $\mathrm{C}_{60}$ in PCBM $\left(\mathrm{C}_{60}\right)$, and (iv) the functional group $\left(\mathrm{C}_{12} \mathrm{H}_{14} \mathrm{O}_{2}\right)$ in PCBM (FG). Based on previous studies, the intermolecular bonds found in the present study can be classified into three types: hydrogen-hydrogen bonds ( $H \cdots H$ ); hydrogen-X bonds ( $H \cdots X$; $\mathrm{X}=\mathrm{C}, \mathrm{O}, \mathrm{S}$ ); and stacking bonds $(C \cdots C$, and $S \cdots Y ; \mathrm{Y}=\mathrm{C}, \mathrm{O})$ as defined previously [34-36]. According to the values of the density and its Laplacian at the interatomic BCPs, and on the bond 
lengths shown in Table 1, QTAIM predicts that none of the interatomic bonds can be considered covalent (shared interactions) rather all are of the closed-shell type since all are characterized by a small value of the electron density at the BCP ( $\rho_{b}<0.01$ a.u.), a positive value of the Laplacian of the electron density at the $\operatorname{BCP}\left(\nabla^{2} \rho_{b}>0\right)$, and an internuclear distance that is generally (significantly) longer than the sum of the van der Waals' radii of the atoms sharing a given bond path. [44-50] We further remark that the charge transfer in the great majority (over 97\%) of these interactions is very small with magnitudes of electric monopoles of the order of $q(\Omega) \sim 10^{-3}$ a.u., that is they are all electro-neutral closed-shell bonding interactions. These values are all consistent with weak to very weak bonding of $\sim 0.5-5 \mathrm{kcal} / \mathrm{mol}$ per interaction, but their sheer number renders their overall effect on the structural stability of the complex non-negligible.

As can be seen from Table 2, $H \cdots X$ bonds are the most frequent bonds in both isomers (64.9\% and $67.5 \%$ of the total number of intermolecular bonds for I1 and I2, respectively). The $H \cdots H$ bonds are the second most frequent interactions $(27.0 \%$ and $17.5 \%$ for I1 and I2, respectively). The least important contribution is the stacking bonding ( $8.1 \%$ and $15.0 \%$ for $\mathrm{I} 1$ and I2, respectively). Thus the noncovalent interactions involving hydrogen atoms account for $91.8 \%$ and $85.0 \%$ of the total number of intermolecular interactions for I1 and I2, while the stacking bonds accounts only for $8.1 \%$ and $15 \%$ for $\mathrm{I} 1$ and I2, respectively. Notice that the $H \cdots X$ bonds are formed mostly between the hydrogen atoms of the hexyl $\left(\mathrm{C}_{6} \mathrm{H}_{13}\right)$ side chains of P3HT and carbons of $\mathrm{C}_{60}$ for both dimer isomers (Table 2). Notice also that all the $H \cdots H$ bonds are formed between one atom on the P3HT side-chains and one on the functional group of PCBM for both isomers. Actually the P3HT side-chains accounts for $83.8 \%$ and $75.0 \%$ of the total number of intermolecular noncovalent bonds, as noncovalent interactions involving hydrogen atoms, in dimer Isomer 1 and Isomer 2, respectively (see Table 2). According to Table 2, the number of bonds with one atom on the $\mathrm{C}_{12} \mathrm{H}_{14} \mathrm{O}_{2}$ 
functional group represent $37.8 \%$ and $42.5 \%$ of the total number of intermolecular bonds for $\quad$ I1 and I2, respectively.

Most of the $H \cdots H$ bonds that occur between the two monomeric units (I1: 8 out of 10; I2: 6 out of 7) are very slightly heteropolar (atomic charges magnitudes $<\sim 10^{-3}$ a.u.) and hence fall close to the hydrogen-hydrogen bonding end of the continuum that has dihydrogen bonding at its other end. These interactions are, thus, not primarily driven by electrostatic atomic multipolar terms between oppositely-charged hydrogens [44-50]. From Table 2, we can see that the stacking bonds, which represent about $10 \%$ in average of the total number of intermolecular bonds, are formed between one atom on the P3HT backbone and one on $\mathrm{C}_{60}$ in isomer I1 (see Table 3 and Figure 3), as might be expected (only three stacking bonds are formed: two $C \cdots C$ and one $C \cdots S$ bonds). However, for I2, besides the four stacking bonds between sulfur atoms on the P3HT backbone and carbon atoms in $\mathrm{C}_{60}$, there are two stacking bonds between atoms in the P3HT side chain and atoms in the PCBM functional group (see Table 3, Figure 3 and related discussion below).

As disscused above and in our previous work, it was reported a break of the global head-tail arrangement between two adjacent rings is broken in the P3HT in-the-dimer I2, as opposed to dimer I1 (see Figure 2). [27] As stated in our previous work [27], this breaking of the translational symmetry in I2 might be due to the relative position of the butyric acid group of PCBM in the dimer. The butyric acid group was supposed to be interacting with nearby P3HT thiophene rings in a stronger way in I2 than in I1. This statement might be confirmed by the present QTAIM analysis. Figure 3 and Table 3 show some of the main interactions of the $\mathrm{C}_{12} \mathrm{H}_{14} \mathrm{O}_{2}$ PCBM functional group with P3HT. First of all, from Table 3 notice that the value of density at any BCP $\left(\rho\left(\overrightarrow{r_{B C P}}\right)\right.$ ) is between $3 \times 10^{-3}$ and $1 \times 10^{-2}$ a.u. with the corresponding value of its Laplacian $\left(\nabla^{2} \rho\left(\overrightarrow{r_{B C P}}\right) \quad\right.$ ) positive. Besides all bond lengths are greater than 2.3 A. Thus, according to QTAIM, all these interactions are closed-shell. [30-33] There are 
three $\quad H \cdots X$ bonds between the $\mathrm{C}_{12} \mathrm{H}_{14} \mathrm{O}_{2}$ functional group FG and P3HT. Notice that, in I1, oxygen O1 participates in two of these three interactions, one with $\mathrm{H} 12$ on the side chain of thiophene ring R8

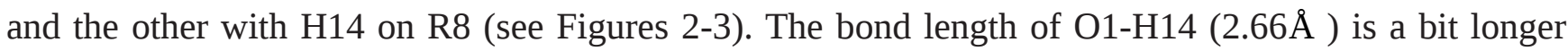

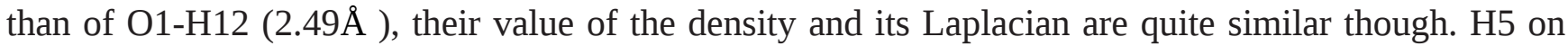
the FG interacts with $\mathrm{C} 17$ on thiophene ring R7 with a bond length equal to 2.73A. The only $\mathrm{H}-\mathrm{H}$ bond is also the shortest one (2.32 $\AA$ ) which is formed between hydrogen H8 on methyl group of PCBM and hydrogen H12 on the side chain of thiophene ring R8. Thus hydrogen H12 undergoes two types of (closed-shell) intermolecular interactions, one $H \cdots X$ type with $\mathrm{O} 1$ and other $H \cdots H$ type with H8. There are three intermolecular stacking interactions in dimer isomer I1 (see Figure 3 and Tables 1 and 3). One is between the sulfur atom S5 on thiophene ring R5 and carbon $\mathrm{C} 22$ on $\mathrm{C}_{60}$, the other two are of type $C \cdots C$. These are the $C 20 \cdots C 21$ and $C 22 \cdots C 23$ noncovalent bonds (see Figure 3), where C20 and C22 are on R4 of P3HT, C21 and C23 are on $\mathrm{C}_{60}$. Notice that none of these stacking bonds involve atoms in the PCBM functional group. However, there is a stacking intramolecular bond between sulfur S8 (of thiophene ring R8) and carbon atom C19 on the side chain of ring R7. As can be seen from Table 3, all stacking bond lengths are greater than $3.1 \AA$ as reported before. [34-35] It is worth mentioning that we decided to show the intramolecular interactions that involve only atoms in the PCBM functional group for the better understanding of the local structural difference between the two P3HT chains in the dimer (see Figure 2 and discussion below).

For the dimer Isomer 2, the intermolecular bonding of the $\mathrm{C}_{12} \mathrm{H}_{14} \mathrm{O}_{2}$ functional group of PCBM is quite different than in Isomer 1. In Isomer 2, the sulfur atom S6 (on thiophene ring R6) undergoes three intermolecular interactions. Two of these are stacking interactions, one is with oxygen $\mathrm{O} 2$ and the other with carbon C6 of PCBM methyl group, with bond length equal to 3.63 and 3.8 $\AA$, respectively. The third interaction of S6 is with hydrogen H3 on the PCBM functional group (see Figure 3 and Table

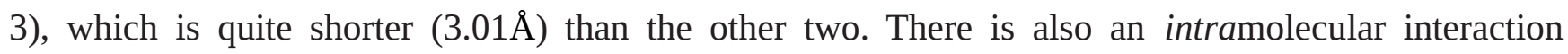




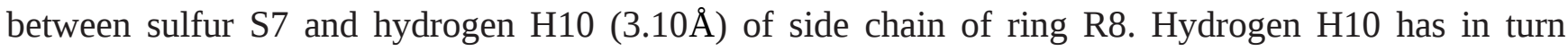
another bond with carbon C9 (2.65A) on the PCBM phenyl group. All other four intermolecular stacking bonds are between one sulfur atom and one carbon atom on $\mathrm{C}_{60}$ (see Table 1 and 3 and Figure 3). Again all intermolecular stacking bond lengths are greater than 3.0 $\AA$ as reported before. [34-35] Thus in dimer Isomer 2 the thiophene rings R6 and R7 participate in more (noncovalent) bonds with nearby atoms than in Isomer 1 . This might explain why thiophene ring R7 in Isomer 2 underwent a higher rotation than the other rings getting in a head-head position with R6 (see Figure 2) breaking so the head-tail symmetry of the P3HT chain. Thus differences in the local bonding between some rings of P3HT and the PCBM C ${ }_{12} \mathrm{H}_{14} \mathrm{O}_{2}$ functional group could explain to some extent the structural differences of the P3HT chains in the dimer. As reported in our original Letter [27], the breaking of the P3HT oligomer chain translational symmetry has repercussion on the dimer absorption spectra. Figure 4 shows the absorption spectra of the P3HT-PCBM dimer isomers (I1 and I2) along with the one of isolated P3HT and PCBM as reported in our original work [27]. If we compare the absorption spectrum of the dimer with the one of the isolated P3HT oligomer, it could be observed an overall blue-shift of the maximum absorption intensity peaks in the dimer with respect to the spectrum of the isolated P3HT oligomer. As discussed above, the translational symmetry (that particular related to the ring head-tail arrangement) was partially broken in P3HT oligomer in Isomer 2 but not in Isomer 1. Thus it could be considered that the ordering destruction of the P3HT chain in the Isomer 2 is more drastic. Coincidentally both the reduction of intensity in the red part and the blue-shift of the maximum absorption intensity peaks of the spectrum are more drastic in the dimer Isomer 2. It is worth mentioning that a blue-shift of the maximum absorption intensity peaks and the reduction of the red part of the absorption spectrum was also experimentally observed for P3HT-PCBM blends which have been attributed to the destruction 
of ordering in the P3HT chains in the presence of PCBM (see the corresponding references in [27]).

\section{Conclusions}

We have performed PBE-TZP-DFT-QTAIM calculations for the two previously reported [27] stable isomers of the supramolecular dimer formed by the 8-unit oligomer of the P3HT and PCBM. A single point PBE-TZP-DFT calculation on each stable dimer isomer -at its optimized geometry- followed by a QTAIM property calculation were performed to "quantify" the contribution of the different chemical groups and different type of chemical bonding to the total intermolecular bonding. According to QTAIM, and as expected, no covalent bonds are present between the P3HT and PCBM in both supramolecular dimer isomers. Noncovalent interactions involving hydrogen atoms (as $H \cdots X$ and $H \cdots H$ bonds) account for $92 \%$ and $85 \%$ of the total number of intermolecular bonds for I1 and I2, while the stacking bonding accounts only for $8 \%$ and $15 \%$ for I1 and I2, respectively. The $H \cdots X$ bonds represent $64.9 \%$ and $67.5 \%$ of the noncovalent interactions for I1 and I2, respectively; the $H \cdots H$ bonds represent $27.0 \%$ and $17.5 \%$, for I1 and I2, respectively.

It was also determined that the $\mathrm{C}_{6} \mathrm{H}_{13}$ side chains of P3HT participate in $83.8 \%$ and $75.0 \%$ of the total number of noncovalent bonds for I1 and I2, respectively; the $\mathrm{C}_{12} \mathrm{H}_{14} \mathrm{O}_{2}$ functional group of PCBM participates in $37.8 \%$ and $42.5 \%$ of the bonds.

In dimer isomer I2, it was shown that the thiophene rings R6 and R7 undergo a stronger chemical bonding with nearby atoms than in isomer I1. This might explain why thiophene ring R7 in I2 underwent a higher rotation than the other rings getting in a head-head position with R6 breaking so the head-tail symmetry of the P3HT chain as reported previously. [27] These structural differences have repercussion on the P3HT-PCBM dimer optical properties. 


\section{Acknowledgments}

JIRH and AWG acknowledge support by UC-MEXUS CONACYT (15-1462). JIR thanks SIP-IPN (project 20151432) for financial support. BMB is thankful for the computer facilities of the "Dirección General de Cómputo y Tecnologías de Información y Comunicación” (DGTIC, UNAM) and the support by PAPIIT-UNAM (IN119811). AWG acknowledges support by the Department of Energy SciDAC program (DE-AC36-99GO-10337) and the National Science Foundation (CHE-1416571). This work used the Extreme Science and Engineering Discovery Environment (XSEDE), which is supported by National Science Foundation grant number ACI-1053575 (award TG-CHE130010 to AWG). CFM acknowledges the Natural Sciences and Engineering Research Council of Canada (NSERC), Canada Foundation for Innovation (CFI), and Mount Saint Vincent University for funding. EAU gratefully acknowledge financial support from Universidad Santo Tomás (Projects FODEIN2013-82, FODEIN-2014-1 and FODEIN-2015-110000101-004). 


\section{REFERENCES}

1. G. Li, R. Zhu, Y. Yang, Nature Photonics 6, 153 (2012).

2. S. S. Sun, N. S. Sariciftci (Eds.), Organic Photovoltaics. Mechanisms, Materials, and Devices, Taylor \& Francis Group LLC, Florida (2005).

3. H. Hoppe, N. S. Sariciftci, "Bulk Heterojunction Solar Cells", in "Organic Photovoltaics. Mechanisms, Materials, and Devices.” S. S. Sun, N. S. Sariciftci (Eds.), Taylor \& Francis Group LLC, Florida (2005).

4. C. Waldauf, P. Schilinsky, J. Hauch and C. J. Brabec, Thin Solid Films, 451, 503 (2004).

5. A. F. Hepp, S. G. Bailey, R. P. Raffaelle in Organic Photovoltaics. Mechanisms, Materials, and Devices. S. S. Sun, N. S. Sariciftci (Eds.), Taylor \& Francis Group LLC, Florida (2005).

6. M. C. Scharber, D. Muhlbacher, M. Koppe, P. Denk, C. Waldauf, A. J. Heeger, C. J. Brabec, Adv. Mater. 18, 789 (2006).

7. J. Brédas, J. E. Norton, J. Cornil, V. Coropceanu, Acc. Chem. Res. 42, 1691 (2009).

8. L. J. K. Koster, V. D. Mihailetchi, P. W. M. Blom, App. Phys. Lett. 88, 093511 (2006).

9. D. L. Cheung, and A. Troisi, J. Phys. Chem. C 114, 20479 (2010).

10. C. F. N. Marchiori, M. Koehler, Syn. Met. 160, 643 (2010).

11. J. J. M. Halls, J. Cornil, D. A. dos Santos, R. Silbey, D. H. Hwang, A. B. Holmes, J. L. Brédas, R. H. Friend, Phys. Rev. B 60, 5721 (1999).

12. S. Cook, R. Katoh, and A. Furube, J. Phys. Chem. C 113, 2547 (2009).

13. T. Drori, C.-X. Sheng, A. Ndobe, S. Singh, J. Holt, Z. V. Vardeny, Phys. Rev. Lett. 101, 037401 (2008).

14. E. Lioudakis, I. Alexandrou, A. Othonos, Nanoscale Res. Lett. 4, 1475 (2009).

15. G. Grancini, D. Polli, D. Fazzi, J. Cabanillas-Gonzalez, G. Cerullo, G. Lanzani, J. Phys. Chem. Lett. 2, 1099 (2011).

16. P. Vanlaeke. A. Swinnen, I. Haeldermans, G. Vanhoyland, T. Aernouts, D. Cheyns, C. Deibel, J. D'Haen, P. Heremans, J. Poortmans, J. V. Manca, Sol. Energy Mater. Sol. Cells 90, 2150 (2006).

17. Y. Kim, S. Cook, S. M. Tuladhar, S. A. Choulis, J. Nelson, J. R. Durrant, D. C. Bradley, M. Giles, I. McCulloch, C. Ha, M. Ree, Nature 5, 197 (2006).

18. B. Peng, Xia Guo, Y. C. Pan, Y. Li, J. Phys. D: Appl. Phys. 44, 365101 (2011). 
19. E. D. Gomez, K. P. Barteu, H. Wang, M. F. Toney, Y. Loo, Chem. Commun. 47, 436 (2011).

20. E. Bundgaard, F. C. Krebs, Sol. Energy Mat. Sol. Cells 91, 954 (2007).

21. J. Zhao, A. Swinnen, G. Van Assche, J. Manca, D. Vanderzande, B. Van Mele, J. Phys. Chem. B 113, 1587 (2009).

22. K. Inoue, R. Ulbricht, P. C. Madakasira, W. M. Sampson, S. Lee, Jose Gutierrez, J. Ferraris, A. Zakhidov, Syn. Met. 154, 41 (2005).

23. G. Janssen, A. Aguirre, E. Goovaerts, P. Vanlaeke, J. Poortmans, J. Manca, Eur. Phys. J. Appl. Phys. 37, 287 (2007).

24. K. Vakhshouri, S. VajjalaKesava, D. R.Kozub, E. D.Gomez, Mater. Lett. 90,97 (2013).

25. W. J. D. Beenken, F. Herrmann, M. Presselt, H. Hoppe, S. Shokhovets, G. Gobsch, E. Runge , Phys. Chem. Chem. Phys. 15, 16494 (2013).

26. T. Liu, A. Troisi, J. Phys. Chem. C 115, 2406 (2011).

27. I. Gutiérrez-González, B. Molina-Brito, A. W. Götz, F.L. Castillo-Alvarado, and J. I. Rodríguez. Chem. Phys. Lett. 612, 234 (2014).

28. S. Grimme, J. Anthony, T. Schwabe, K. Muck-Lichtenfeld, Org. Biomol. Chem. 5, 741 (2007).

29. S. Grimme, J. Anthony, S. Ehrlich, H. Krieg, J. Chem. Phys. 132, 154104 (2010).

30. R. F. W. Bader, Atoms in Molecules: A Quantum Theory. Oxford University Press (1994).

31. C. F. Matta, R. J. Boyd (Eds.) The Quantum Theory of Atoms in Molecules. From Solid State to DNA and Drug Design; Wiley-VCH: Weinheim (2007).

32. C. F. Matta, J. Hernandez-Trujillo, T. H. Tang, R. F. W. Bader, Chem. Eur. J. 9, 1940 (2003).

33. C.F. Matta, N. Castillo, R.J. Boyd J. Phys. Chem. B 110, 563 (2006).

34. O. A. Zhikol, O. V. Shishkin, K. A: Lyssenko, J. Leszczynski, J. Chem. Phys. 122, 144104 (2005).

35. M. Waller, A. Robertazzi, J. Platts, D. Hibbs, P. Williams, J. Comp. Chem. 27, 4 (2006).

36. R. Parthasarathi, V. Subramaian, Sruct. Chem. 16, 243 (2005).

37. J. I. Rodríguez, R. F. W. Bader, P. W. Ayers, C. Michel, A. Götz, C. Bo, Chem. Phys. Lett. 472, 149 (2009). 
38. J. I. Rodríguez, J. Comp. Chem. 34, 681 (2013).

39. ADF 2014. E. J. Baerends, J. Autschbach, J. A. Berger, A. Berces, F. M. Bickelhaupt,C. Bo, P. L. de Boeij, P. M. Boerrigter, L. Cavallo, D. P. Chong, L.Deng, R. M. Dickson, D. E. Ellis, M. van Faassen, L. Fan, T. H. Fischer, C.Fonseca Guerra, S. J. A. van Gisbergen, A. W. Götz, J. A. Groeneveld, O. V.Gritsenko, M. Gru“ ning, F. E. Harris, P. van den Hoek, C. R. Jacob, H. Jacobsen,L. Jensen, E. S. Kadantsev, G. van Kessel, R. Klooster, F. Kootstra, M.V. Krykunov, E. van Lenthe, J. N. Louwen, D. A. McCormack, A. Michalak,J. Neugebauer, V. P. Nicu, V. P. Osinga, S. Patchkovskii, P. H. T. Philipsen, D. Post, C. C. Pye, W. Ravenek, J. I. Rodríguez, P. Romaniello, P. Ros, P. R. T. Schipper, G. Schreckenbach, J. G. Snijders, M. Sola, M. Swart, D. Swerhone, G. te Velde, P. Vernooijs, L. Versluis, L. Visscher, O. Visser, F. Wang, T. A. Wesolowski, E. M. van Wezenbeek, G. Wiesenekker, S. K. Wolff, T. K. Woo, A. L. Yakovlev, and T. Ziegler. ADF 2013. Theoretical Chemistry, Vrije Universiteit: Amsterdam, The Netherlands. Available at: http://www.scm.com. Acessed on April 7, 2014.

40. C. F. Guerra, J. G. Snijders, G. te Velde, E. J. Baerends, Theor. Chem. Acc. 99, 391 (1998).

41. G. te Velde, F. M. Bickelhaupt, S. J. A. van Gisbergen, C. Fonseca Guerra, E. J. Baerends, J. G. Snijders, T. Ziegler, J. Comput. Chem. 22, 931 (2001).

42. J. P. Perdew, K. Burke, M. Ernzerhof, Phys. Rev. Lett. 77, 3865 (1996).

43. J. Antony, S. Grimme, D. G. Liakos, F. Neese, J. Phys. Chem. A 115, 11210 (2011).

44. V.I. Bakhmutov, “Dihydrogen Bonds: Principles, Experiments, and Applications”. WileyInterscience, New Jersey, (2008).

45. R.H. Crabtree, Acc. Chem. Res. 23, 95 (1990).

46. P.L.A. Popelier, J. Phys. Chem. A 102, 1873 (1998).

47. M.J. Calhorda, Chem. Commun. 801 (2000).

48. S.J. Grabowski, J. Phys. Chem. A 104, 5551 (2000).

49. J. Echeverría, G. Aullón, D. Danovich, S. Shaik and S. Alvarez, Nature Chemistry 3, 323 (2011).

50. E. A. Uribe, M. C. Daza, J. L. Villaveces, Chem. Phys. Lett. 490, 143 (2010). 
Figure 1. a) The optimized structures of the P3HT-PCBM dimer for isomer 1 (upper row) and isomer 2 (lower row) as obtained in our original work. [27] b) the same optimized structures as in a) but now hiding the alkane side chains in P3HT and hydrogen atoms. Isomer 1 is the lowest-energy isomer. The energy difference between the two isomers is equal to $3.13 \mathrm{eV}$.

a)
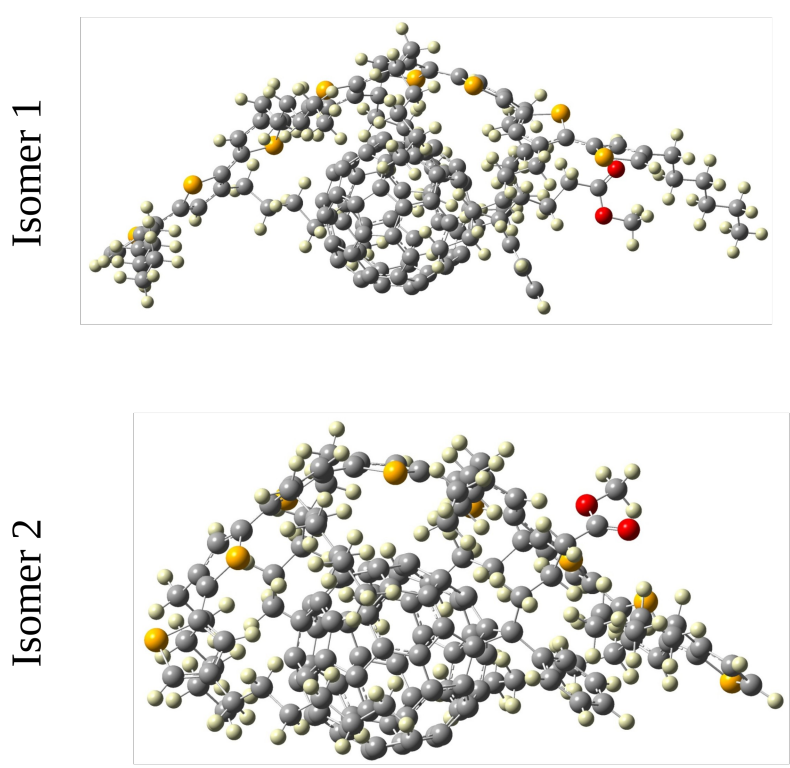

b)
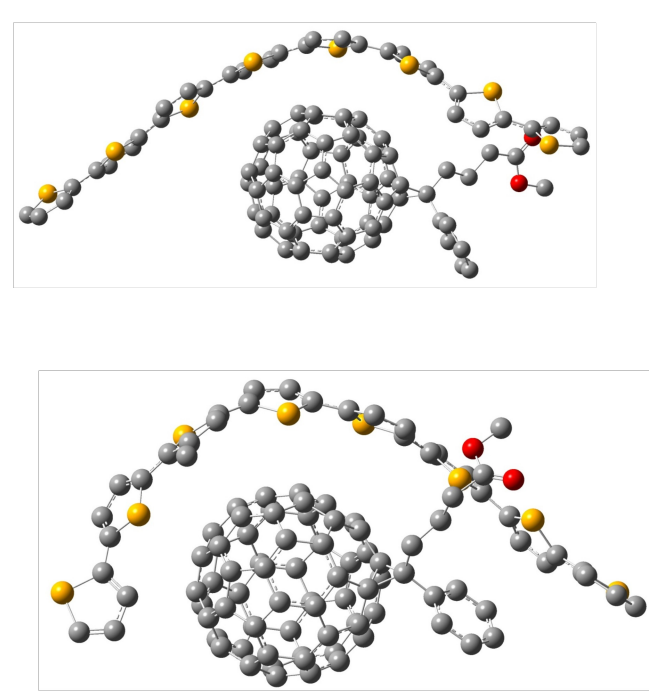
Figure 2. Structures with the dihedral angle $\left(\mathrm{D}_{\mathrm{i}}\right)$ values in degrees between adjacent thiophene rings of P3HT in-the-dimer for (a) Isomer 1, and (b) Isomer 2.

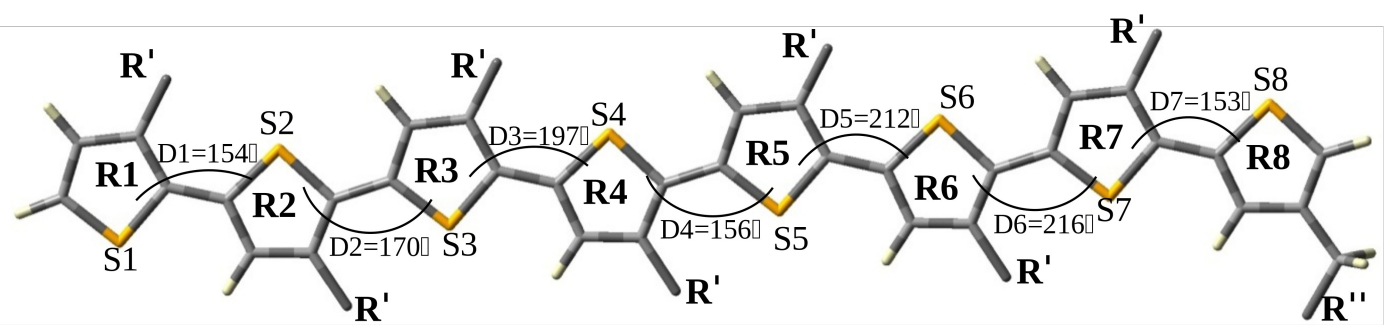

a)

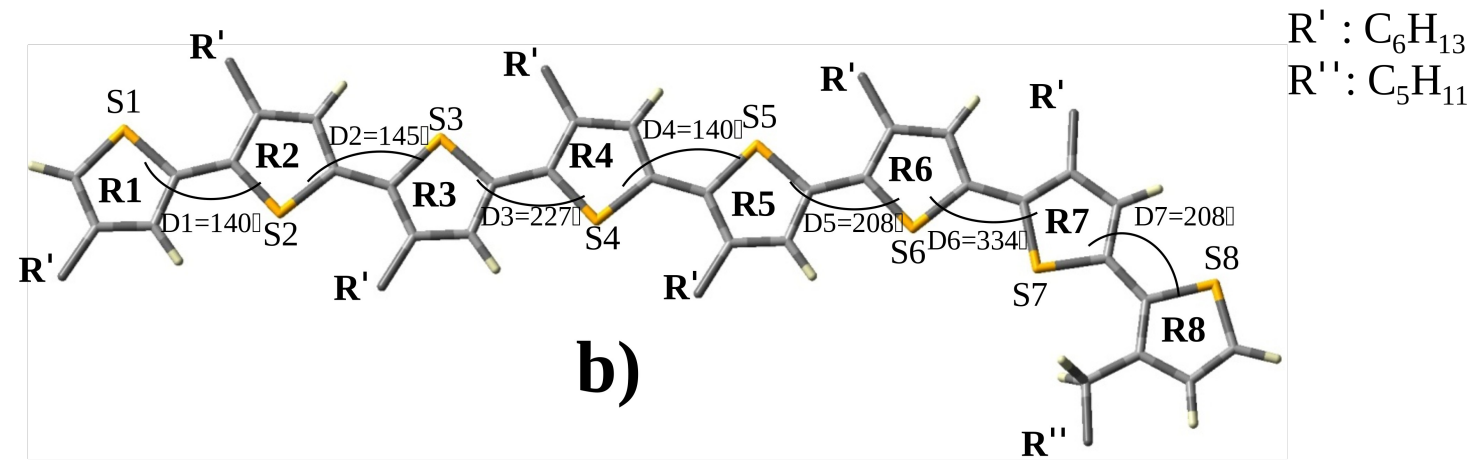


Figure 3. Intermolecular stacking (orange), H-X (blue) and H-H (brown) interactions. Intramolecular stacking interactions are also shown (green). a) Isomer 1, b) Isomer 2. The inset shows details of the PCBM functional group in a different orientation for clarity. Bond information of these interactions is listed in Table 3.
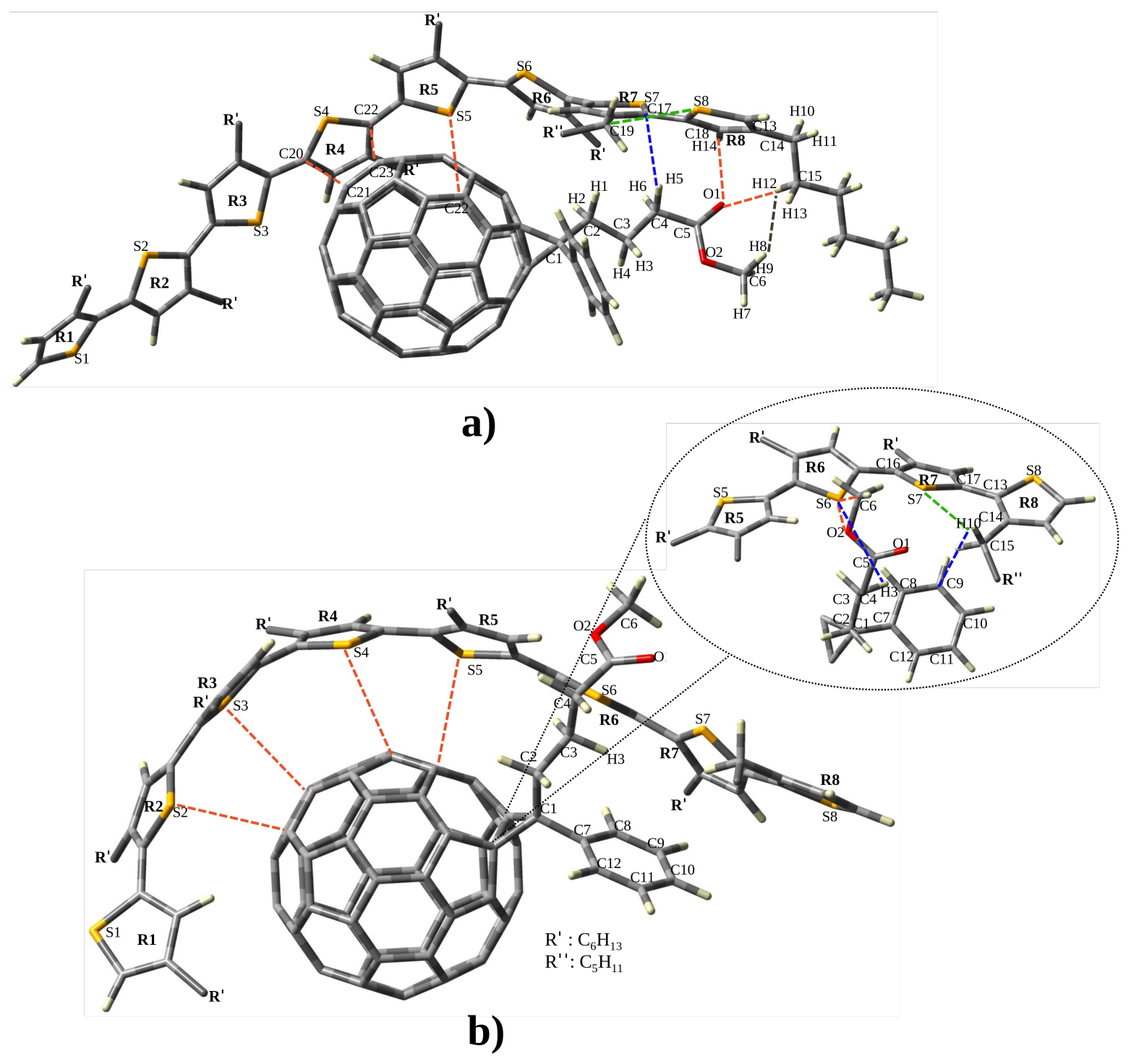
Figure 4 (Reproduction from reference [27]). Absorption spectra for the isolated P3HT (red line), isolated PCBM (black line), P3HT-PCBM dimer Isomer 1 (green line) and Isomer 2 (blue line) obtained via TDDFT calculations at the DFT optimized geometries. The absorption spectrum of isolated PCBM can be seen in more detail in the inset.

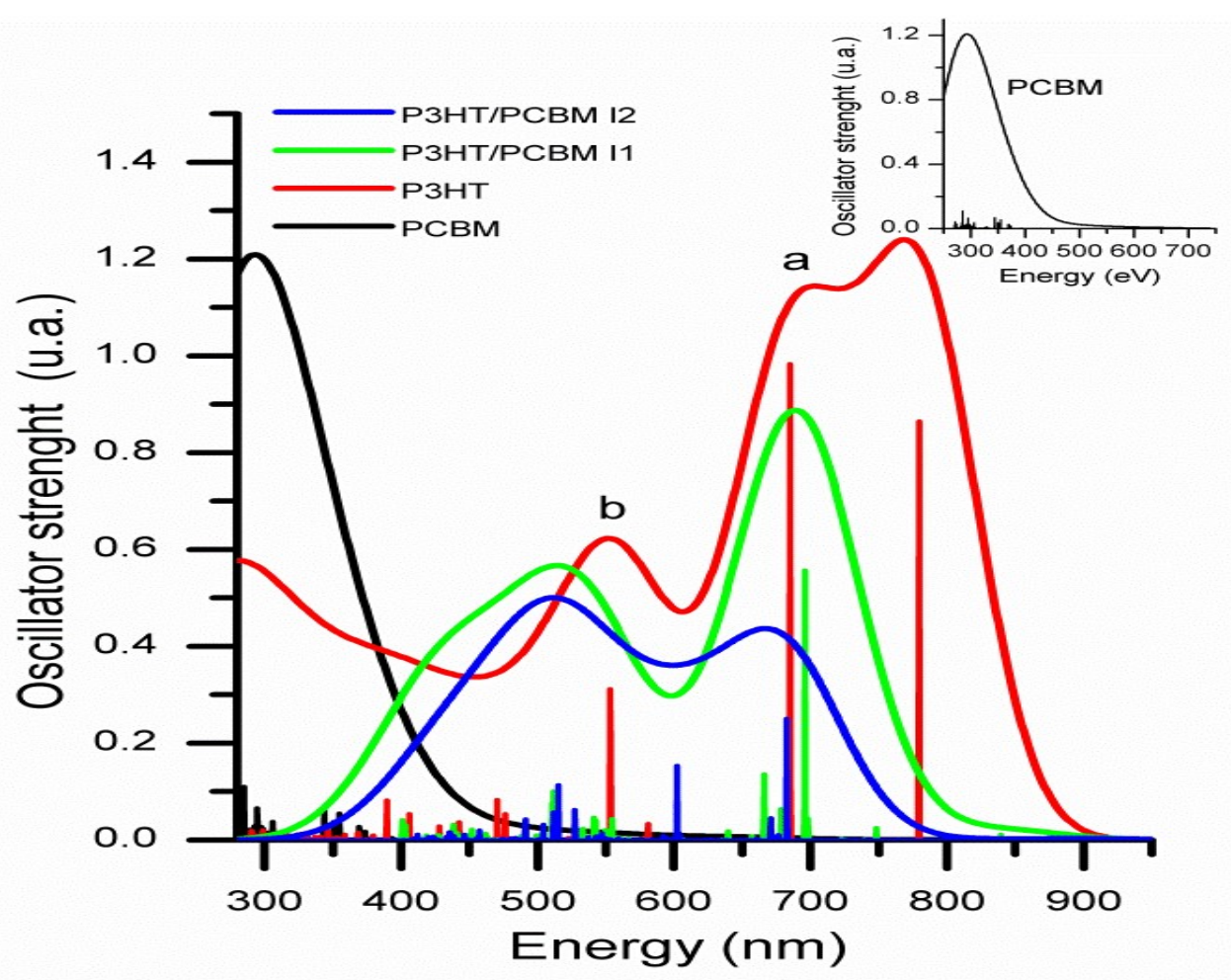


Table 1. Minimum (min), maximum (max) and average (aver) values (in a.u.) of the electron density $\rho$ and its Laplacian $L=\nabla^{2} \rho$ at each type of intermolecular BCPs. Bond length (d) values (in $\AA$ ) are also shown. Last row shows the global value of each quantity over the total BCPs of each type.

\section{Isomer I1}

\begin{tabular}{|c|c|c|c|c|c|c|c|c|c|}
\cline { 2 - 10 } \multicolumn{1}{c|}{} & \multicolumn{3}{c|}{$\rho$} & \multicolumn{2}{c|}{ L } & \multicolumn{3}{c|}{ d } \\
\hline Bond Type & $\min$ & $\max$ & aver & $\min$ & $\max$ & aver & $\min$ & $\max$ & aver \\
\hline H...X & 0.0035 & 0.0107 & 0.0065 & 0.011 & 0.032 & 0.020 & 2.50 & 3.21 & 2.81 \\
\hline H...H & 0.0024 & 0.0100 & 0.0069 & 0.007 & 0.037 & 0.023 & 2.09 & 2.75 & 2.30 \\
\hline STACKING & 0.0076 & 0.0117 & 0.0090 & 0.023 & 0.033 & 0.027 & 3.01 & 3.47 & 3.24 \\
\hline Total Global & 0.0024 & 0.0117 & 0.0074 & 0.007 & 0.037 & 0.024 & 2.09 & 3.47 & 2.79 \\
\hline
\end{tabular}

\section{Isomer I2}

\begin{tabular}{|c|c|c|c|c|c|c|c|c|c|}
\hline & \multicolumn{3}{|c|}{$\rho$} & \multicolumn{3}{|c|}{ L } & \multicolumn{3}{|c|}{ d } \\
\hline Bond Type & $\min$ & $\max$ & aver & $\min$ & $\max$ & aver & $\min$ & $\max$ & aver \\
\hline H...X & 0.0025 & 0.0103 & 0.0046 & 0.008 & 0.039 & 0.015 & 2.36 & 3.34 & 3.01 \\
\hline H...H & 0.0028 & 0.0063 & 0.0041 & 0.010 & 0.021 & 0.013 & 2.27 & 2.72 & 2.50 \\
\hline STACKING & 0.0035 & 0.0075 & 0.0054 & 0.013 & 0.022 & 0.016 & 3.47 & 3.80 & 3.60 \\
\hline Total Global & 0.0025 & 0.0103 & 0.0047 & 0.008 & 0.039 & 0.015 & 2.27 & 3.80 & 3.03 \\
\hline
\end{tabular}


Table 2. Number of different types of intermolecular BCPs between atoms of different chemical groups: P3HT $\mathrm{C}_{6} \mathrm{H}_{13}$ side chains (SC), the P3HT backbone (BB), the $\mathrm{C}_{60}$ in PCBM ( $\mathrm{C}_{60}$ ), and the functional group ( $\mathrm{C}_{12} \mathrm{H}_{14} \mathrm{O}_{2}$ ) in PCBM (FG). Last row shows the percentage contribution of each intermolecular group interaction. Last column shows the percentage contribution of each type of intermolecular BCPs.

Isomer 1 (I1)

\begin{tabular}{|c|c|c|c|c|c|c|} 
Bond Type & SC - C60 & SC - FG & BB - C60 & BB - FG & P3HT-PCBM & Percentage \\
\hline H...X & 20 & 1 & 0 & 3 & 24 & $64.86 \%$ \\
\hline H...H & 0 & 10 & 0 & 0 & 10 & $27.03 \%$ \\
\hline STACKING & 0 & 0 & 3 & 0 & 3 & $8.11 \%$ \\
\hline Total & 20 & 11 & 3 & 3 & 37 & $100.00 \%$ \\
\hline Percentage & $54.05 \%$ & $29.73 \%$ & $8.11 \%$ & $8.11 \%$ & $100.00 \%$ & \\
\hline
\end{tabular}

Isomer 2 (I2)

\begin{tabular}{|c|c|c|c|c|c|c|}
\hline Bond Type & SC - C60 & SC - FG & BB - C60 & BB - FG & P3HT-PCBM & Percentage \\
\hline H...X & 18 & 5 & 1 & 3 & 27 & $67.50 \%$ \\
\hline H...H & 0 & 7 & 0 & 0 & 7 & $17.50 \%$ \\
\hline STACKING & 0 & 0 & 4 & 2 & 6 & $15.00 \%$ \\
\hline Total & 18 & 12 & 5 & 5 & 40 & $100.00 \%$ \\
\hline Percentage & $45.00 \%$ & $30.00 \%$ & $12.50 \%$ & $12.50 \%$ & $100.00 \%$ & \\
\hline
\end{tabular}


Table 3. Value fo the electron density $\rho$, its Laplacian $L=\nabla^{2} \rho$, bond length (d) and QTAIM bond path (BP Length) length. All these interactions are presented graphically in Figure 3.

\begin{tabular}{|c|c|c|c|c|c|}
\hline \multicolumn{6}{|c|}{ Isomer 1} \\
\hline \multicolumn{2}{|c|}{ Interactions } & $\mathrm{d}(\AA)$ & BP Lenth $(\AA)$ & $\rho(\mathrm{r})$ (a.u.) & $\mathrm{L}=\nabla^{2} \rho$ (a.u.) \\
\hline C...H & C17...H5 & 2.733 & 2.872 & 0.007 & 0.023 \\
\hline \multirow{2}{*}{ O...H } & O1...H14 & 2.665 & 2.704 & 0.006 & 0.022 \\
\hline & $\mathrm{O} 1 \ldots \mathrm{H} 12$ & 2.497 & 2.505 & 0.008 & 0.029 \\
\hline H...H & H8...H12 & 2.324 & 2.409 & 0.006 & 0.021 \\
\hline \multirow{2}{*}{ S...C } & S8...C19* & 3.224 & 3.593 & 0.010 & 0.035 \\
\hline & S5...C22** & 3.465 & 3.548 & 0.008 & 0.024 \\
\hline \multirow{2}{*}{ С...C } & C20...C21** & 3.251 & 3.607 & 0.008 & 0.023 \\
\hline & $\mathrm{C} 22 \ldots \mathrm{C} 23 * *$ & 3.010 & 3.039 & 0.012 & 0.033 \\
\hline
\end{tabular}

\begin{tabular}{|c|c|c|c|c|c|}
\hline \multicolumn{6}{|c|}{ Isomer 2} \\
\hline C...H & С9...Н10 & 2.654 & 2.72 & 0.013 & 0.041 \\
\hline \multirow{2}{*}{ S...H } & S6...H3 & 3.012 & 3.042 & 0.006 & 0.018 \\
\hline & S7...H10* & 3.103 & 3.157 & 0.004 & 0.012 \\
\hline S...O & S6...O2 & 3.615 & 3.621 & 0.004 & 0.015 \\
\hline \multirow{5}{*}{ S...C } & S6...C6 & 3.801 & 4.013 & 0.003 & 0.013 \\
\hline & S2 ...C** & 3.673 & 3.677 & 0.005 & 0.014 \\
\hline & S3... $C^{* *}$ & 3.523 & 3.527 & 0.006 & 0.017 \\
\hline & S4.... $C^{* *}$ & 3.467 & 3.581 & 0.007 & 0.022 \\
\hline & S5... $C^{* *}$ & 3.503 & 3.505 & 0.006 & 0.018 \\
\hline
\end{tabular}

\section{* Intramolecular stacking interactions \\ ** Backbone- $\mathrm{C}_{60}$ stacking interactions}

